\title{
Quantum Antenna Theory for Secure Wireless Communications
}

\author{
Said Mikki*, \\ *Dept. of ECECS, University of New Haven, West Haven, CT, USA, said.m.mikki@gmail.com*
}

\begin{abstract}
We provide a very broad outline for a new research area within the domain of Future Antennas, namely quantum antenna (q-antenna) theory and their applications to building secure digital communication lines. The proposed quantum antenna theory purports to presenting a natural extension of RF antennas in classical wireless to the now established field of quantum communications. The paper provides a bird's eye view on the subject, highlighting the main themes and the expected results and benefits of such research domain.
\end{abstract}

Index Terms-Future antennas, quantum communications, cybersecurity.

\section{INTRODUCTION}

Quantum communications (QC) is now an established frontier in high-tech Research \& Development all around the world, though most of this research is currently being conducted by universities and specialized quantum institutes affiliated with the academic sector [1]-[5]. One of their main advantages is the fact that quantum cryptography provides a means to build unbreakable and fully-protected secure communication lines (this is based on the celebrated no-cloning theorem - valid only in the quantum regime - and other results, see [3].) Given the ongoing progress in fundamental experiments verifying theoretical predictions about the feasibility of long-distance quantum telecommunications (QTC), it is expected, that quantum technology may enter into mainstream industry within the near future by establishing commercial mass production of sources, receivers, routers, and repeaters pertaining to quantum networks. Quantum communication (q-communications) differs from classical communication (ccommunications) by the mode through which information is physically processed and transmitted. In classical communication links, information is transmitted by means of aggregates of fundamental identical particle-carriers, e.g., electrons, photons, polaritons, etc, where the main "size" or "strength" of the information signal is here measured by the energy of the carrier, which is an extensive quantity like length, volume, and mass [6]-[12]. In contrast, quantum communications relies on the pure quantum state as the main physical carrier of information [1]. Quantum states, whether pure or mixed, are much more general - and hence more abstract - physical structures than electromagnetic fields and electrons [13], [14]. A quantum state does not necessarily require the electromagnetic field itself to be modified in order to carry information. In fact, the electromagnetic field is only one possible physical observable operator of the quantum state among others. This makes quantum communications richer in content and latent

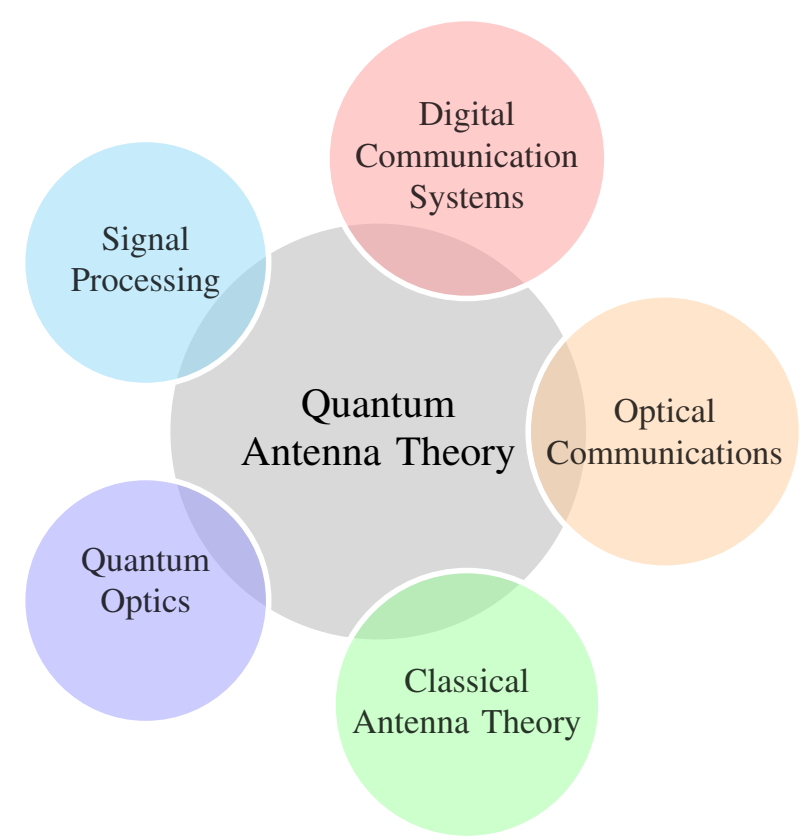

Fig. 1: The interdisciplinary nature of the proposed quantum antenna theory.

potentials due to the peculiar non-classical structure. Many of the researches conducted throughout the last decade in the area of quantum communication receiver design have attempted to explicitly exploit this inherent richness of the quantum state by systematically searching (mainly through optimization methods) for best "measurement operators" in order to extract the maximum possible information about of information from the transmitted quantum state with good efficiency [1]. It seems that less effort has been put on searching for physical realization of quantum states and the physics inherent in the process of radiation or state production. The goal of the present research is attempting to build a physico-engineering theory for wireless quantum communications. In order to achieve this, we propose dividing the program outlined here into two main components:

1) Quantum Antenna Theory.

2) Digital Communication Transceiver Design.

The present text will focus on giving some outline pertaining to the first component, while the paper [15] elaborates the second point. This research has evolved from considerations related to the use of semi-classical methods in solving problems 
at the nano- and meso-scale [16]-[22] to the more complete program of second quantization [23], [24] of quantum field theory. It was also further motivated by the realization that a momentum-space approach is needed to deal with radiation and propagation problems in nonlocal metamaterials [25][30], a technical fact that will play a role in the quantum antenna theory proposed here. A multidisciplinary approach such as this is rarely addressed in the applied electromagnetic community, into which antenna engineering is embedded. However, the author believes there is a need to upgrade the semi-classical approach of nanotechnology to directly treat potential engineering applications where the concept of photon is essential. This paper is an outline of such a possible research program for the special case of antenna theory.

\section{Classical vs Quantum Antenna Theories}

What is meant by the quantum antenna (hereafter, qantenna) theory? In order to better appreciate the peculiar nature of the new research field here proposed, it may help to first review the concept of antenna as an engineering device, after which the fundamental distinction between classical and quantum can be formally given. Antennas are man-made devices created for the purpose of converting energy electromagnetic form from confined modes to open, unconfined, radiations modes that can be transmitted into unbounded domains surrounding the antenna [6], [10]. In other words, an antenna is an energy conversion device with a very specific sense. Rather than being a system that can convert one genre of energy to another, for example transforming chemical energy into electricity (battery), a classical antenna converts the same type of energy, the electromagnetic, from one mode of operation into another [10]. The common mode of the electromagnetic energy exciting the antenna is waveguide/transmission line mode, the guided-wave mode, where energy tends to flow in one direction only, the longitudinal or axial orientation of the waveguide structures supporting the wave [31]. On the other hand, the antenna, if successfully operating, will convert this guided mode energy that is localized at the antenna input port into un-localized, that is, radiation mode capable of traveling far away from the source that produced it [10] (the source producing these modes being localized at the antenna's physical body itself and the near-field rim zone [27].) For quantum antennas, however, the physical process is fundamentally different from the one described above. The information source is still a localized current distribution $\mathbf{J}(\mathbf{r}, t)$ as before, but the final outcome of the radiation process is no longer a localized radiation in one position or direction (e.g., far field in a certain beamwidth), but rather a global quantum state, which we denote $|\Phi\rangle$. Fig. 2 illustrates this process where we notice that the q-state is always produced by passing through the radiation zone of the antenna. The major operational modes of $\mathrm{c}$ - and q- antennas, especially in light of recent research in classical applied electromagnetic theory, are explained in Fig. 3. Relevant to the global nature of the radiation state in q-antennas is the formal fact that $|\Phi\rangle$ does not dependent on position. Indeed, in the Schrodinger picture,

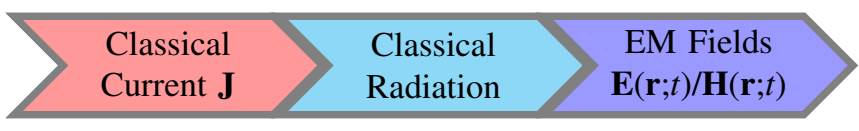

(a) Classical antennas

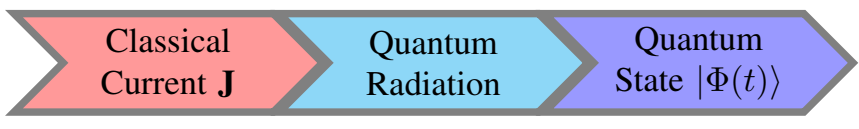

(b) Quantum antennas

Fig. 2: The general structure of the the antenna physical transformations.

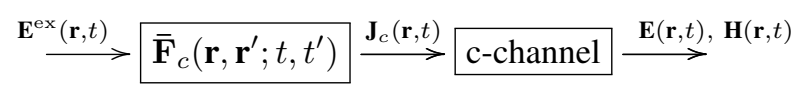

(a) Classical antennas.

$$
\left.\stackrel{\mathbf{E}^{\mathrm{ex}}(\mathbf{r}, t)}{\longrightarrow} \overline{\mathbf{F}}_{q}\left(\mathbf{r}, \mathbf{r}^{\prime} ; t, t^{\prime}\right) \stackrel{\mathbf{J}_{q}(\mathbf{r}, t)}{\longrightarrow} \underset{\text { q-channel }}{\longrightarrow} \longrightarrow \alpha(t)\right\rangle
$$

(b) Quantum antennas.

Fig. 3: The fundamental operational modes of c- and q- antennas. Both types requires a current Green's function (ACGF) $\overline{\mathbf{F}}$ [10] to connect classical excitation field $\mathbf{E}^{\mathrm{ex}}$ with a classical induced current $\mathbf{J}$. Note that in both c- and q- antennas, the induced currents $\mathbf{J}_{c}$ and $\mathbf{J}_{q}$, respectively, are classical. The difference, however, resides mainly in the "output" or the radiation state, which is classical (quantum) in c- (q-) antennas.

the q-antenna $|\Phi(t)\rangle$ evolves with time while the observables are function of position only. In the Heisenberg picture (the framework we will often adopt in our theory besides the interaction picture), the observables become functions of both position $\mathbf{r}$ and time $t$, e.g., the electric field operator $\mathbf{E}(\mathbf{r}, t)$ or the photon number operator $N(\mathbf{r}, t)$.

\section{Coherent State Radiation by Quantum ANTENNAS}

It has been shown as early as the beginnings of the 1960s that a classical current sufficiently isolated from the back-reaction of the quantum radiation will produce pure coherent states [13]. A coherent state, also sometimes known as Glauber's state though they were discovered nearly forty years earlier by Schrodinger in the 1920 s, can be represented mathematically by the simple form $|\alpha\rangle$, where $\alpha \in \mathbb{C}$ is a complex number. This state can be expanded in terms of the standard Fock's $n$-state of photon radiation $|n\rangle, n=1,2, \ldots$, (exactly $n$ light quanta excited) via the expansion [13]

$$
|\alpha\rangle=e^{-\frac{1}{2}|\alpha|^{2}} \sum_{n=0}^{\infty} \frac{\alpha^{n}}{\sqrt{n !}}|n\rangle .
$$

Further properties of coherent states have been developed very early in its history and can be found in excellent texts like [14], 


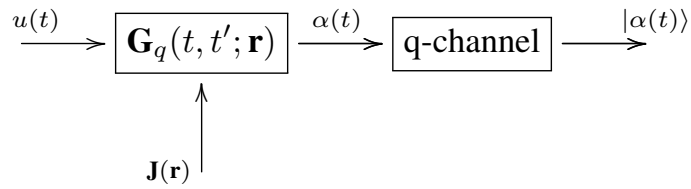

Fig. 4: The fundamental structure of the q-antenna problem. The q-Green's function $\mathbf{G}_{q}\left(t, t^{\prime} ; \mathbf{r}\right)$ represents the linearsystem-like properties of the antenna. The transformation from the complex state $\alpha(t)$ to the quantum radiation state $|\alpha(t)\rangle$, however, is nonlinear. Details of this derivation and other indepth analytical aspects of radiation in q-antennas will be provided by the author in the expanded version of this paper.

[32]. By means of this expansion, one can effectively reduce computations with coherent states to calculations with Fock's state, an approach we will adopt for applications to quantum wireless communication systems. It is also possible to use coherent states themselves as basis to expand every other state since such states are known to be complete (in fact, they are overcomplete, see [14].) The proof that classical currents produce coherent states is not widely discussed in literature, but often appears in quantum optics textbooks like [14], [32] and more advanced quantum mechanics works such as [33]. However, to our best knowledge, no proposal for developing a comprehensive theory of quantum antennas has been advanced so far. Indeed, optical antennas for instance, a topic now widely popular, is not actually an example of what we mean here by q-antennas. It appears that quantum optics favors more the approach of free fields where the details of the current producing radiation are ignored. This is quite understandable from the historical perspective since the invention of laser and its rise in the late 1960s shifted research toward quantum optics and especially investigations related to coherence and quantum correlation [13], [32].

\section{M-ary Digital Communications Utilizing QUANTUM ANTENNAS}

Helstrom's classical study includes consideration of digital and analog quantum communication schemes [1]. The discussion in that book focused more on the implementation of the receiver (via an aperture-coupled resonance cavity followed by measurement at time samples.) However, even with the recent revival of the topic, still there is no comprehensive investigation regarding how to generate the transmitted states of the q-telecommunication link. This is not surprising given that a quantum antenna theory has not been developed so far, though the required tools like Glauber's theory and array signal processing are available. One of the major applications of the proposed q-antenna theory is to provide a direct path toward building applications for quantum digital wireless communication link design, especially along the lines of the q-MIMO architectures proposed in [15].

In general, for an M-ary digital communication link, one is interested in sending $M$ symbols encoded by $M$ different

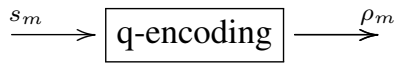

Fig. 5: The process of mapping $M$ classical symbols to $M$ $\mathrm{q}$-states in q-communications using a specially designed $\mathrm{q}$ antenna.

states $\rho_{1}, \rho_{2}, \ldots, \rho_{M}$, where each $\rho$ is the density operator of the state. This process is termed here 'q-encoding' and is captured by Fig. 5. Consider a multimode coherent radiation state $|\bar{\alpha}\rangle$ given by

$$
|\bar{\alpha}\rangle=\prod_{\mathbf{k} s}\left|\alpha_{\mathbf{k} s}\right\rangle,
$$

where the product state is formed over all wavevectors $\mathbf{k}$ and polarizations $s=1,2$. Here, we have

$$
\left|\alpha_{\mathbf{k} s}\right\rangle=e^{-\frac{1}{2}\left|\alpha_{\mathbf{k} s}\right|^{2}} \sum_{n=0}^{\infty} \frac{\alpha^{n}}{\sqrt{n !}}\left|n_{\mathbf{k} s}\right\rangle .
$$

Digital information may be formed via a quantum encoding scheme in which each complex number $\alpha_{\mathbf{k} s}$ is assigned one symbol. That is, in a finite dimensional approximation of (2) in $M$ dimension, we can write

$$
|\bar{\alpha}\rangle=\left|\alpha_{1}\right\rangle \otimes\left|\alpha_{2}\right\rangle \otimes \cdots \otimes\left|\alpha_{M}\right\rangle,
$$

where $\alpha_{m} \in \mathbb{C}, m=1,2, \ldots, M$, is the complex amplitude of the coherent state representation of the $m$ th mode. On the other hand, q-antenna theory will provide an alternative, more sophisticated method to form the q-encoding mapping of Fig. 5. The idea is to utilize the newly derived q-antenna Green's function in Fig. 4 to directly engineer the spectral (moementum) representation of the multimode coherent state produced by the q-antenna. The major key findings are the following:

1) The radiated $\mathbf{q}$-state $\mathbf{k} s$-structure can be directly linked to the spatio-temporal functional form of the classical excitation current distribution $\mathbf{J}(\mathbf{r}, t)$.

2) Classical array theory can be linked to the q-antenna Green's function $\mathbf{G}_{q}$ in order to devise special algorithms allowing engineering the q-encoding mapping from classical information to q-states.

3) Quantum antenna theory allows us to better understand how spatial considerations in the Tx and Rx sides of the q-communication links affects coding and reception of information sent through a q-channel. These considerations can be applied to the proposed q-MIMO scheme of quantum digital wireless transmission.

The major idea here is that q-antenna theory permits us to go beyond the simple approach based on the expansion (4) because in our theory the exact form of the relation between 1) geometry of the q-antenna and 2) the spatio-temporal function of the excitation are both taken into account simultaneously. It will be shown that one can form a virtually unlimited qspace constellations for digital quantum communications by carefully altering the physical layout and/or excitations of 


\begin{tabular}{|c|c|c|}
\hline $\begin{array}{c}\text { Classical } \\
\text { spatio- } \\
\text { pulse } \\
\text { stream } \\
u(t)\end{array} \rightarrow \quad \begin{array}{c}\text { Desired } \\
\text { q-state }|\alpha\rangle \\
\text { current } \\
\mathbf{J}(\mathbf{r}) u(t)\end{array} \rightarrow \begin{array}{c}\text { for digital } \\
\text { commu- } \\
\text { nication }\end{array}$ \\
\hline
\end{tabular}

Fig. 6: The design process of q-antennas for digital communication applications. The user will generate a given sequence of classical pulses $u(t)$ which in turns are used to feed an optical antenna. When the antenna is excited, a spatio-temporal current $\mathbf{J}(\mathbf{r}) u(t)$ is produced. The proposed q-antenna theory aims at explicating how the temporal and spatial forms of the classical current source can be manipulated to engineer the internal momentum-space structure of the radiated q-state, which in turn can be exploited for building optimum M-ary quantum wireless communication links.

the Tx q-antenna system, which in our case will be first approached using ideas borrowed from classical antenna theory then expanded into new domains and functions conductive to the new research area of quantum antenna theory.

\section{CONCLUSION}

In our opinion, the main advantage of q-antenna theory is that it allows a deeper understanding of both classical and quantum channels since coherent states, the bases of the proposed framework, provide a minimum-uncertainty quantum solution of radiation problems; i.e., it is theoretically the nearest a quantum state can get close to classical electromagnetic radiation states. Therefore, a smooth transition from classical antenna theory to the new research area of quantum antenna theory can be effected by means of the analytical results available through this work. A numerical scheme to implement the formalism will be developed where spectral integrals over all modes will be computed. Optimization theory can then be integrated with the implementation to devise optimum radiating q-antenna layouts capable of generating desired q-signal space constellations to serve in present and future quantum communication systems (see Fig. 6.)

\section{REFERENCES}

[1] C. W. Helstrom, Quantum detection and estimation theory. Academic Press: Cambridge University Press, 1976.

[2] G. Cariolaro, Quantum communications. Springer, 2016.

[3] M. Nielsen and I. L. Chuang, Quantum computation and quantum information. Cambridge New York: Cambridge University Press, 2010.

[4] M. Wilde, Quantum information theory. Cambridge, UK New York: Cambridge University Press, 2017.

[5] G. Benenti, G. Casati, D. Rossini, and G. Strini, Principles of quantum computation and information: a comprehensive textbook. Singapore Hackensack, NJ: World Scientific Publishing Co. Pte. Ltd, 2019.

[6] S. A. Schelkunoff and H. T. Friss, Antennas: Theory and practice. New York; Chapman \& Hall: London, 1952.

[7] S. Mikki and Y. Antar, "On the Fundamental Relationship Between the Transmitting and Receiving Modes of General Antenna Systems: A New Approach," IEEE Antennas and Wireless Propagation Letters, vol. 11, pp. 232-235, 2012.

[8] - "The antenna current Green's function formalism-Part I," IEEE Trans. Antennas Propagat, vol. 9, pp. 4493-4504, September 2013.
[9] — "The antenna current Green's function formalism-Part II," IEEE Trans. Antennas Propagat, vol. 9, pp. 4505-4519, September 2013.

[10] —-, New Foundations for Applied Electromagnetics: The Spatial Structure of Fields. London: Artech House, 2016.

[11] —, "A rigorous approach to mutual coupling in general antenna systems through perturbation theory," IEEE Antennas and Wireless Communication Letters, vol. 14, pp. 115-118, 2015.

[12] S. M. Mikki and Y. M. M. Antar, "A new technique for the analysis of energy coupling and exchange in general antenna systems," IEEE Transactions on Antennas and Propagation, vol. 63, no. 12, pp. 55365547, Dec 2015.

[13] R. Glauber, Quantum theory of optical coherence: selected papers and lectures. Weinheim: Wiley-VCH, 2007.

[14] J. Klauder and E. C. G. Sudarshan, Fundamentals of quantum optics. Mineola, N.Y: Dover Publications, 2006.

[15] S. Mikki, "A quantum MIMO architecture for antenna wireless digital communications," Progress In Electromagnetics Research C, vol. 93, pp. 143-156, 2019.

[16] S. Mikki and A. Kishk, "Effective medium theory for carbon nanotube composites and their potential applications as metamaterials," in 2007 IEEE/MTT-S International Microwave Symposium, June 2007, pp. 1137 1140.

[17] — "Mean-field electrodynamic theory of aligned carbon nanotube composites," IEEE Transactions on Antennas and Propagation, vol. 57, no. 5, pp. 1412-1419, May 2009.

[18] S. M. Mikki and A. A. Kishk, "Electromagnetic scattering by multi-wall carbon nanotubes using effective-boundary condition approach," in 2008 IEEE Antennas and Propagation Society International Symposium, July 2008, pp. 1-4.

[19] S. Mikki and A. Kishk, "Electromagnetic scattering by multi-wall carbon nanotubes," Progress In Electromagnetics Research B, vol. 17, pp. 4967, 2009.

[20] — "A symmetry-based formalism for the electrodynamics of nanotubes," Progress In Electromagnetics Research, vol. 86, pp. 111-134, 2008.

[21] S. M. Mikki and A. A. Kishk, "Exact derivation of the dyadic green's functions of carbon nanotubes using microscopic theory," in 2007 IEEE Antennas and Propagation Society International Symposium, June 2007, pp. 4332-4335.

[22] S. Mikki and A. Kishk, "Theory of optical scattering by carbon nanotubes," Microwave and Optical Technology Letters, vol. 49, no. 10, pp. 2360-2364, Jul. 2007.

[23] E. Zeidler, Quantum field theory I: Basics in Mathematics and Physics. Springer, 2009.

[24] — Quantum field theory II: Quantum Electrodynamics. Springer, 2006.

[25] K. Cho, Optical response of nanostructures: microscopic nonlocal theory. Berlin New York: Springer, 2003.

[26] — Reconstruction of macroscopic Maxwell equations: a single susceptibility theory. Berlin, Germany: Springer, 2018.

[27] O. Keller, Quantum Theory of Near-Field Electrodynamics. Berlin New York: Springer, 2011.

[28] S. Mikki and A. Kishk, "Electromagnetic wave propagation in nonlocal media: Negative group velocity and beyond," Progress In Electromagnetics Research B, vol. 14, pp. 149-174, 2009.

[29] S. Mikki and Y. Antar, "On electromagnetic radiation in nonlocal environments: Steps toward a theory of near field engineering," in 2015 9th European Conference on Antennas and Propagation (EuCAP), April 2015, pp. 1-5.

[30] S. Mikki and A. Kishk, "Nonlocal electromagnetic media: A paradigm for material engineering," in Passive Microwave Components and Antennas. InTech, April 2010.

[31] J. Schwinger et al., Classical electrodynamics. Reading, Mass: Perseus Books, 1998.

[32] J. C. Garrison and R. Chiao, Quantum optics. Oxford: Oxford University Press, 2014

[33] E. Merzbacher, Quantum mechanics. New York: Wiley, 1998. 